\title{
Comparison of Doppler waveform and mean velocity of hepatic vein in cirrhotic patients with healthy subjects and their correlation with severity of cirrhosis.
}

\author{
Islam $\mathbf{M}^{1}$, Ahmed $\mathrm{AU}^{2}$, Mohiuddin $\mathrm{AS}^{3}$
}

\begin{abstract}
This descriptive study was carried out in the Department of Radiology and Imaging, BIRDEM (Bangladesh Institute of Research and Rehabilitation in Diabetes, Endocrine and Metabolic Disorder) Dhaka during the period of 1st June 2008 to 30th May 2009, to compare the pattern of the Doppler waveform and mean velocity of hepatic vein between healthy subjects and that in cirrhotic patients and to detect possible differences and also to assess the accuracy of the detection of changes in hepatic vein waveform and mean velocity for the diagnosis of liver cirrhosis. A total of 40 cirrhotic patients and 40 healthy subjects were enrolled in this study. Doppler waveform was done in all the patients and the healthy subjects. The mean hepatic vein velocity (MHV) was $12.22 \pm 4.22 \mathrm{~cm} / \mathrm{sec}$ and $5.17 \pm 1.19 \mathrm{~cm} / \mathrm{sec}$ in case and healthy group respectively. The mean MHW difference was significantly $(p<0.001)$ higher in case group. $37.5 \%$ patient had wave form HV- 0 and HV-1 and $25.0 \%$ had wave form HV-2 in case group, however, in healthy group all subjects had wave form HV- 0 and the difference was statistically significant $(p<0.05)$ between case and healthy. In Child's class A wave form HV-0 was found in $75.0 \%$, HV-1 was $25.0 \%$ and HV-2 was not found in case group. In Child's class B wave form HV-0 was found in $21.4 \%, \mathrm{HV}-1$ was $71.5 \%$ and $\mathrm{HV}-2$ was $7.1 \%$. In Child's class $\mathrm{C}$ wave form HV-0 was not found, $\mathrm{HV}-1$ was $10.0 \%$ and $\mathrm{HV}-2$ was $90.0 \%$. The and the difference was statistically significant $(p<0.05)$ among the different grading level with wave form. In Child's class $A$ the mean velocity was $9.18 \pm 1.81 \mathrm{~cm} / \mathrm{sec}$ and velocity ranged from 7.5 to $14.2 \mathrm{~cm} / \mathrm{sec}$. In Child's class B the mean velocity was $11.26 \pm 1.81 \mathrm{~cm} / \mathrm{sec}$ and velocity ranged from 7.5 to $14.2 \mathrm{~cm} / \mathrm{sec}$. In Child's class $C$ the mean velocity was $18.44 \pm 1.91 \mathrm{~cm} / \mathrm{sec}$ and velocity ranged from 15.5 to $20.9 \mathrm{~cm} / \mathrm{sec}$. The mean velocity was statistically significant $(p<0.05)$ among Child's class $A$, Child's class B and Child's class C. From the above summary and previous study it is observed that there is a significant difference between Doppler waveform and mean velocity of right hepatic vein in cirrhotic patients and in normal subjects. It can be used as a new parameter in the assessment of liver cirrhosis.
\end{abstract}

CBMJ 2014 July: Vol. 03 No. 02 P: 23-28

Keywords: Hepatic vein; Hepatic vein velocity; Doppler ultrasound.

\section{Introduction}

The diagnosis of liver cirrhosis is important in the evaluation and management of patients. Liver biopsy is the gold standard but it is invasive. Ultrasonography is a non-invasive and useful modality in assessing the liver for certain conditions but low frequency ultrasonography is not a sensitive test for the diagnosis of liver cirrhosis in daily clinical practice ${ }^{1}$.

In liver cirrhosis there are changes in liver parenchyma as well as alteration of hepatic vasculature, including morphologic changes of hepatic veins $(\mathrm{HVs})^{2}$. It is accepted that abnormal circulation caused by hyper dynamic circulation and intrahepatic shunt persists in cirrhotic liver ${ }^{2}$. Although above alterations of these vessels are already known, normal and pathologic aspects of Doppler waveform ${ }^{3}$ and mean velocity of HVs have been less studied².

Doppler waveform of the hepatic veins in healthy human is a triphasic waveform,

1. ${ }^{*}$ Dr. Mahzabeen Istam

Assistant professor Radiology \& Imaging,

Community Based Medical College, Bangladesh.

2 Dr.Akhter Uddin Ahmed

Ex-Head of the Department, Radiology \& Imaging, BIRDEM, Dhaka, Bangladesh.

3. Dr.A.S. Mohiuddin

Head of the Department, Radiology \& Imaging,

BIREDM, Dhaka, Bangladesh.

"Address of correspondence

Email :akashovan@yahoo.co.uk

Mobile: 01711269017 
containing two negative waves and one positive wave ${ }^{4}$. As for abnormal hemodynamics of the hepatic veins in cirrhotic liver reported and suggested 5,6 that the Doppler waveform becomes flat and mean velocity changes when hepatocellular function is impaired ${ }^{2}$. Some studies even proposed that flattening of the hepatic wave form and altered mean velocity of $\mathrm{HVs}$ could be used as a diagnostic tool for liver cirrhosis ${ }^{2,3,7}$.

This study compared the Doppler ultrasonographic wave pattern and mean velocity of hepatic veins in a group of patients affected by liver cirrhosis with a group of healthy subjects comparable for sex and age. This was done to detect possible differences in hepatic vein waveform and mean velocity in these two groups and investigate the relationship of these differences with the severity of disease according to Child-Pugh scoring ${ }^{8}$ and to assess the accuracy of the detection of changes in hepatic vein waveform and mean velocity for the diagnosis of liver cirrhosis.

\section{Methods}

This descriptive study was carried out on total of 83 subjects (both cirrhotic patients and healthy adults, cirrhotic and healthy subjects being 43 and 40 respectively. The case group was admitted to the Gastro hepatic and pancreatic department (GHPD) of BIRDEM and diagnosed as cirrhotics. All of them were referred in the department of Radiology and Imaging of BIRDEM and Doppler examination was done from $1^{\text {st }}$ June, 2008 to $30^{\text {th }}$ May, 2009. They were routinely investigated using appropriate haematological and biochemical parameters to come to the clinical diagnosis. The patients were then referred for sonographic evaluation for the diagnosis of cirrhosis and Doppler examination was done. Consecutively 43 cirrhotic patients were taken in case group. Among the 43 cirrhotic patients 3 patients were excluded from the case group due to presence of nodule of HCC. Finally 40 patients were included in the case group. There were 32 male and 8 female patients in case group and 27 male and 13 female in normal healthy subjects. Normal healthy subjects were those people who came to the Radiology \& Imaging department of BIRDEM for other complaints and sonographically no abnormality was detected. Relevant data for the scoring of the severity of the cirrhosis were collected from the patients' record.

Doppler ultrasonographic examination was performed by means of duplex ultrasonographic equipment with a convex probe of $3.5 \mathrm{MHz}$ provided by a pulsed Doppler device operating at frequency of 2.5 $\mathrm{MHz}$. The system was equipped with software to compute the time-averaged velocity from the spectral display after placement of the calipers. Each subject was examined while in a supine position after overnight fasting to avoid any influence of meal and posture on splanchnic hemodynamics. The morphological appearance of the hepatic veins was evaluated by assessing caliber and course. Doppler study was then performed by positioning the same volume, as large as at least one-third of the vessel diameter, within the right hepatic vein and was approached from right intercostals space. The reason behind choosing the right hepatic vein over other was that the middle and left hepatic vein often join each other before draining to the inferior vena cava. In each case the angle of incidence of the Doppler ultrasound beam was maintained between $20^{\circ}$ and $50^{\circ}$, and the spectral analysis was always recorded for at least 4-6 seconds with a filter of $100 \mathrm{~Hz}$. During examination each subject was asked to breathe normally or to just simple holdup of breathing to avoid modification by deep inspiration. Mean flow velocity was calculated automatically by the instrument after tracing of the spectral display.

The Doppler ultrasound wave pattern was then arbitrarily classified into one of three groups according to the Doppler signal characteristics:

1. HVO, triphasic waveform (i.e., presence of a short phase of reverse flow)

2. HV1, decreased amplitude of the phasic oscillations without the short phase of reverse flow. 
3. HV2, completely flat waveform without any phasic oscillation, similar to the Doppler trace of a portal branch.

Finally the severity of liver cirrhosis was assessed by Child-Pugh scoring system.

Choice of ultrasound machine and transducer:

Ultrasound machine:

Siemens Antares Sonoline

Medisone Sono Ace 8000 Live

Transducer:

3.5 Megahertz curvilinear transducer provided by a pulse Doppler device operating at frequently of $2.5 \mathrm{MHz}$

All the relevant collected data were compiled on a master chart first, then organized by using scientific calculator and standard statistical formula. Percentages were calculated to find out the proportion of the findings. Further statistical analyses of the results were done by computer software devised as the statistical package for social scientist (SPSS). The results were presented in Tables, Figures, and Diagrams etc. For significant of differences unpaired't' test and Chi square tests and ANOVA test were applied. A ' $p$ ' value $<0.05$ was considered as significant.

\section{Results}

Mean hepatic vein velocity (MHVV) (cm/sec) of the study subjects in case and healthy $(n=80)$

In case group the mean hepatic vein velocity (MHVV) was $12.22 \mathrm{~cm} / \mathrm{sec}$ with standard deviation of mean (SD) $\pm 4.22 \mathrm{~cm} / \mathrm{sec}$ and their MHVV ranged from 6.2 to $20.9 \mathrm{~cm} / \mathrm{sec}$. However, in healthy group, the mean MHVV was $5.17 \mathrm{~cm} / \mathrm{sec}$ with standard deviation of mean (SD) $\pm 1.19 \mathrm{~cm} / \mathrm{sec}$ and their MHVV ranged from 3.0 to $7.2 \mathrm{~cm} / \mathrm{sec}$. The mean MHVV difference was statistically highly significant $(p<0.001)$ between case and healthy in unpaired t-test. The results are shown in the table I.

Table I: Mean hepatic vein velocity (MHVV) (cm/sec) of the study subjects in case and control $(n=80)$.

\begin{tabular}{|l|c|c|c|c|c|}
\hline MHVV (cm/sec) & \multicolumn{2}{|c|}{$\begin{array}{c}\text { Case } \\
(\mathrm{n}=40)\end{array}$} & \multicolumn{2}{c|}{$\begin{array}{c}\text { Control } \\
(\mathrm{n}=40)\end{array}$} & P value \\
\hline Mean \pm SD & 12.22 & \pm 4.22 & 5.17 & \pm 1.19 & $0.001^{\mathrm{S}}$ \\
\hline Range (Min-max) & $(6.2$ & $-20.9)$ & $(3.0$ & $-7.2)$ & \\
\hline
\end{tabular}

unpaired $\mathrm{t}$ test, $\mathrm{t}$ value $=10.19, \mathrm{df}=78$, $p=0.001, s=$ significant, significant $(p<0.05)$ with unpaired $t$ test.

Right hepatic vein wave form of the study subjects in case and healthy $(n=80)$

The Doppler ultrasound wave pattern in right hepatic vein was classified in three groups according to the Doppler signal. HV-O was the normal triphasic waveform, $\mathrm{HV}-1$ was biphasic waveform and HV-2 was completely flat waveform. The highest percentage of patients (37.5\%) had wave form HV-O and HV-1 and 10 $(25.0 \%)$ had wave form HV-2 in case group, however, in healthy group all subjects had wave form HV-0 and the difference was statistically significant $(p<0.05)$ between case and control in chi square test. The results are shown in the figure $\mathrm{I}$.

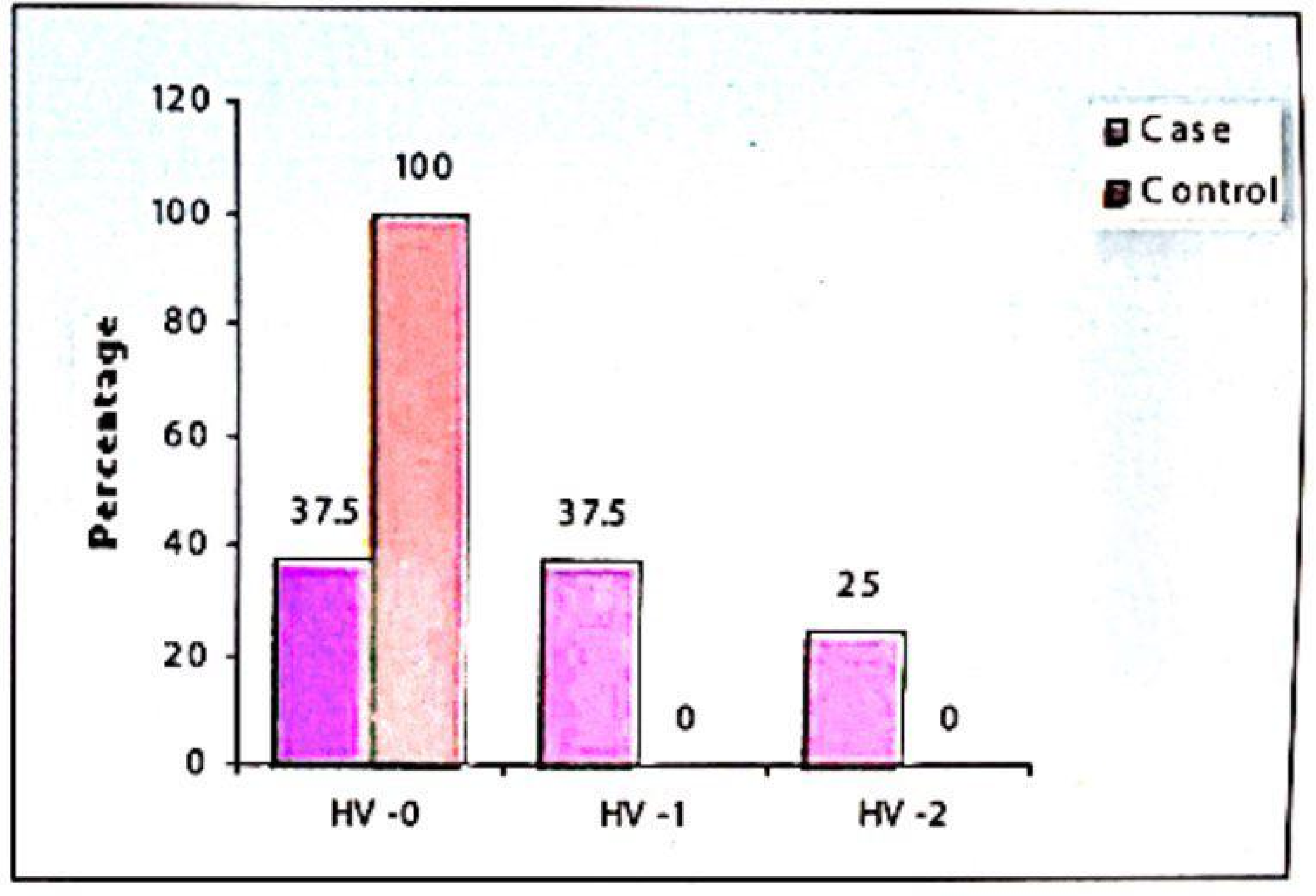

Figure I: Bar diagram showing right hepatic vein wave form of the study subjects in case and healthy $(n=80)$.

Association between Wave form and severity of the liver cirrhosis according to Child-Pugh scoring of the study subjects in case $(n=40)$

The severity of the liver cirrhosis was assessed by Child-Pugh scoring system with the parameters of encephalopathy, serum 
bilirubin, serum albumin, prothombin time and ascites. Adding the individual score $<7$ was Child's class A, 7-9 was Child's class B and > 9 was Child's class $C$. In Child's class A wave form HV-0 was found in 12 (75.0\%), HV-1 was $4(25.0 \%)$ and HV-2 was not found in case group. In Child's class B wave form HV-O was found in $3(21.4 \%), \mathrm{HV}-1$ was $10(71.5 \%)$ and HV-2 was $1(7.1 \%)$. In Child's class $C$ wave form HV-0 was not found, HV-1 was $1(10.0 \%)$ and $\mathrm{HV}-2$ was $9(90.0 \%)$. The difference was statistically significant $(p<0.05)$ among the different grading level with wave form in chi square test. The results are shown in the table II and figure II.

Table II: Association between Wave form and severity of the liver cirrhosis according to Child-Pugh scoring of the study subjects in case $(n=40)$.

\begin{tabular}{|l|c|c|c|c|c|c|c|}
\hline \multirow{2}{*}{$\begin{array}{l}\text { Wave } \\
\text { form }\end{array}$} & \multicolumn{2}{|c|}{ Child's A } & \multicolumn{2}{|c|}{ Child's B } & \multicolumn{2}{|c|}{ Child's C } & \multirow{2}{*}{ P value } \\
\cline { 2 - 7 } & $\mathrm{n}$ & $\%$ & $\mathrm{n}$ & $\%$ & $\mathrm{n}$ & $\%$ & \\
\hline HV-0 & 12 & 75.0 & 3 & 21.4 & 0 & 0.0 & \\
\hline HV-1 & 4 & 25.0 & 10 & 71.5 & 1 & 10.0 & $0.001^{\mathrm{S}}$ \\
\hline HV-2 & 0 & 0.0 & 1 & 7.1 & 9 & 90.0 & \\
\hline
\end{tabular}

Chi square test, chi value $=40.38, d f=4, p=$ $0.001, s=$ significant, significant $(p<0.001)$ with chi square test.

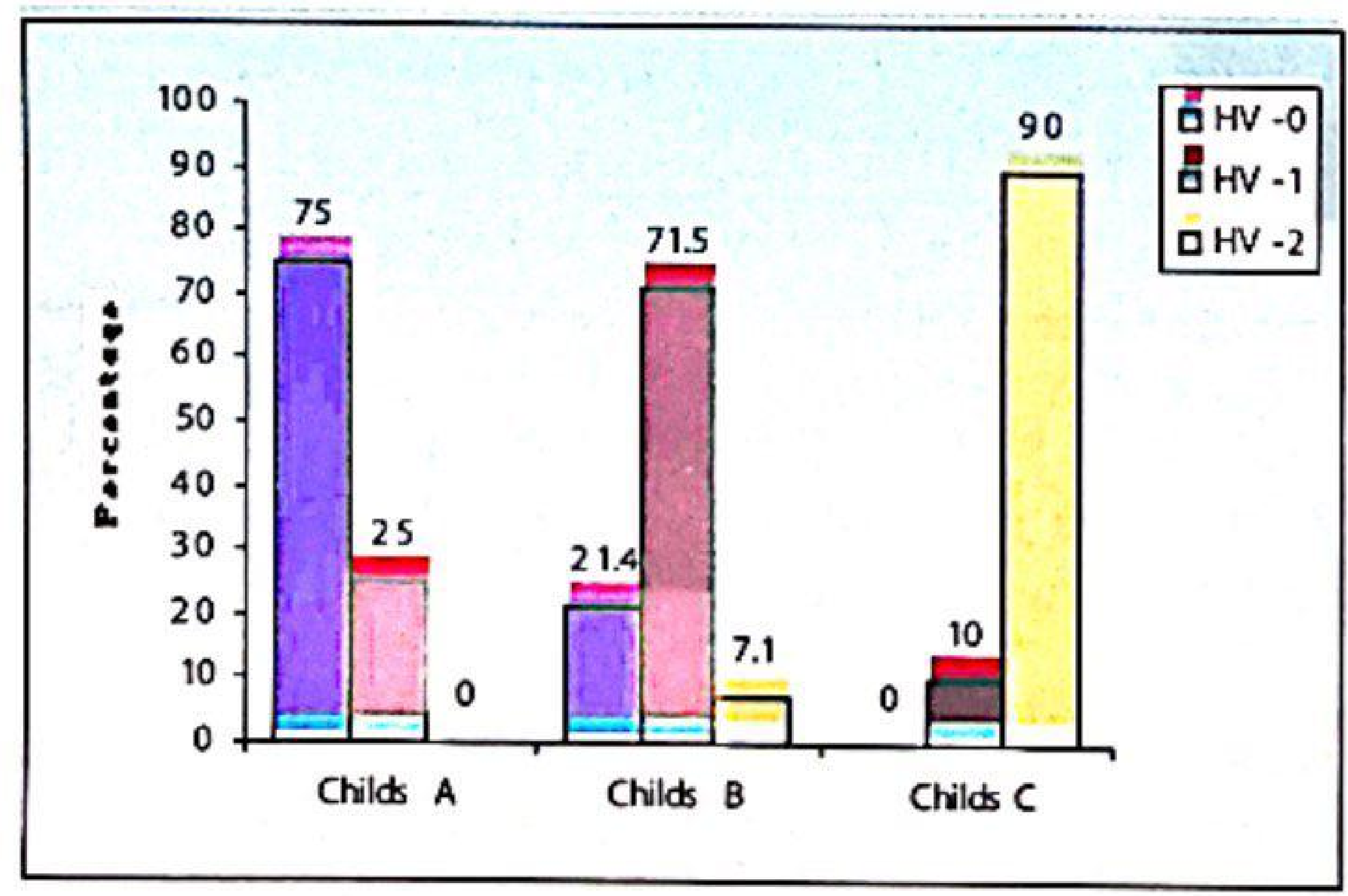

Figure II: Bar diagram showing association between wave form and severity of the liver cirrhosis according to Child-Pugh scoring of the study subjects in case $(n=40)$.

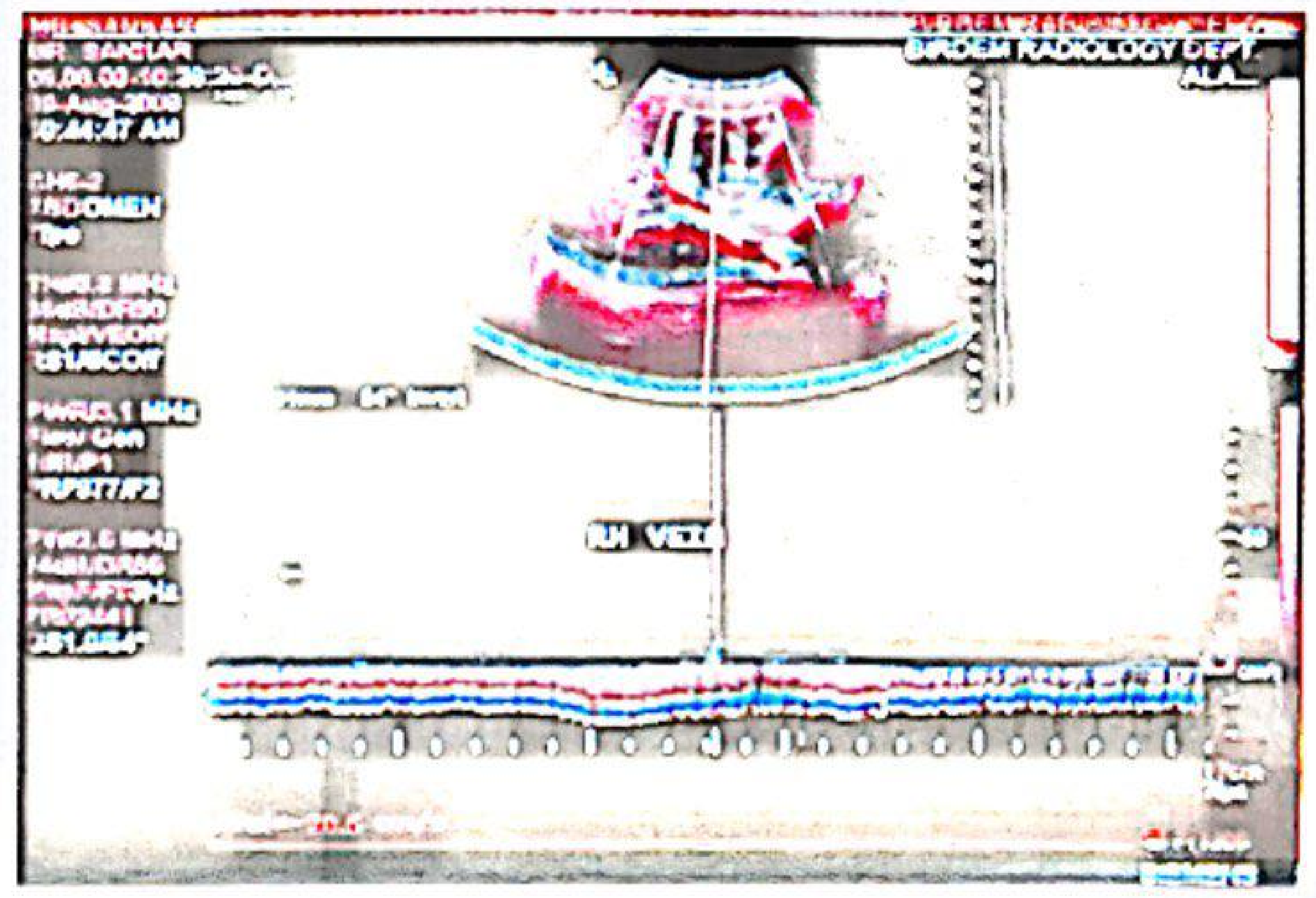

Figure III: Flat Doppler flow patterns of right hepatic vein of a 61 years male patient with liver cirrhosis.

Mean velocity was $20.5 \mathrm{~cm} / \mathrm{sec}$ (case no. 11).

Association between mean hepatic vein velocity and severity of liver cirrhosis according to Child-Pugh scoring of the study subjects in case $(n=40)$

According to Child's class $A$ the mean velocity was $9.18 \mathrm{~cm} / \mathrm{sec}$ with standard deviation of mean (SD) $\pm 2.17 \mathrm{~cm} / \mathrm{sec}$ and their velocity ranged from 6.2 to $15.0 \mathrm{~cm} / \mathrm{sec}$. In Child's class $B$ the mean velocity was $11.26 \mathrm{~cm} / \mathrm{sec}$ with standard deviation of mean (SD) \pm 1.81 $\mathrm{cm} / \mathrm{sec}$ and their velocity ranged from 7.5 to $14.2 \mathrm{~cm} / \mathrm{sec}$. In Child's class $\mathrm{C}$ the mean velocity was $18.44 \mathrm{~cm} / \mathrm{sec}$ with standard deviation of mean (SD) $\pm 1.91 \mathrm{~cm} / \mathrm{sec}$ and their velocity ranged from 15.5 to $20.9 \mathrm{~cm} / \mathrm{sec}$. The mean velocity was statistically significant $(p<0.05)$ among Child's class A, Child's class $B$ and Child's class $C$ in ANOVA test. The results are shown in the table III.

Table III: Association between mean hepatic vein velocity and severity of liver cirrhosis according to Child-Pugh scoring of the study subjects in case $(n=40)$.

\begin{tabular}{|l|c|c|r|r|}
\hline Child-Pugh Scoring & Mean & \pm SD & Range & P value \\
\hline Child's class A & 9.18 & \pm 2.17 & $(6.2-15.0)$ & \\
\hline Child's class B & 11.26 & \pm 1.81 & $(7.5-14.2)$ & $0.001^{\text {s }}$ \\
\hline Child's class C & 18.44 & \pm 1.91 & $(15.5-20.9)$ & \\
\hline
\end{tabular}

ANOVA test, $F$ value $=69.43, d f=2,37, p=$ $0.001, s=$ significant, significant $(p<0.05)$ with ANOVA test. 


\section{Discussion}

Vulnerable to wide variety of metabolic, circulatory, toxic, microbial and neoplastic insults, the liver is the most frequently injured organs in the body and there are various diseases of the liver. Among the diseases of the liver cirrhosis is most common.

The exact prevalence of cirrhosis in world wide is unknown. It is the 9th leading cause of death in USA and etiology is mainly alcohol abuse. Almost same picture is reported in Europe and numbers are even higher in most Asian and African countries where chronic hepatitis B \& C infection is common ${ }^{9}$.

Definitive diagnosis of liver cirrhosis remains a challenging problem for both physician and radiologist, especially in early cases, as many remain symptom less. Often a poor correlation exists between histologic findings and the clinical picture. Cirrhosis can lead to many complications, among them hepato cellular carcinoma (HCC) is the dangerous one. Early diagnosis, staging and regular follow up of the cirrhosis are important for the proper treatment to prevent or minimize complications of cirrhosis.

A rational selection and combination of diagnostic imaging modalities such as ultrasonography and pulsed Doppler examination of hepatic vein are essential for the detection and evaluation of cirrhosis at an early stage to prevent different complications, progression of the disease and regular follow up.

This case healthy study was carried out with an objective to compare the pattern of the Doppler waveform and mean velocity of hepatic vein between healthy subjects and that in cirrhotic patients and also to detect possible differences and to assess the accuracy of the detection of changes in hepatic vein waveform and mean velocity for the diagnosis and severity of liver cirrhosis.

Sudhamshu et $a^{2}$ found in their study that the mean hepatic vein velocity was $12.7 \pm 6.4$ $\mathrm{cm} / \mathrm{sec}$ and $6.2 \pm 3.2 \mathrm{~cm} / \mathrm{sec}$ in cirrhotics and non cirrhotics group respectively, which is significantly higher in cirrhotic patients.
Similarly, Herbay et al. ${ }^{6}$ observed identical mean hepatic vein velocity between patient with liver disease and healthy group. It closely resemble with the present study where the mean hepatic vein velocity (MHVV) was $12.22 \pm 4.22 \mathrm{~cm} / \mathrm{sec}$ with the range from 6.2 to $20.9 \mathrm{~cm} / \mathrm{sec}$. In control group, the mean MHVV was $5.17 \pm 1.19 \mathrm{~cm} / \mathrm{sec}$ with the range from 3.0 to $7.2 \mathrm{~cm} / \mathrm{sec}$. The mean MHVV difference was significantly $(p<0.001)$ higher in case group in this study.

In this study the Doppler ultrasound wave pattern in right hepatic vein was classified in three groups according to the Doppler signal. $\mathrm{HV}-\mathrm{O}$ was the normal triphagic waveform, HV1 was biphasic waveform and $\mathrm{HV}-2$ is completely flat waveform. It was observed that more than one third $(37.5 \%)$ of patients had wave form HV-0 (normal triphagic waveform) \& HV-1 (biphasic waveform) and $25.0 \%$ had wave form HV-2 (completely flat waveform) in case group. In control group all subjects had wave form HV-O (normal triphagic waveform) and the HV-0 was significantly $(p<0.05)$ higher in control group. Sudhamshu et al. ${ }^{2}$ reported all non-cirrhotic control showed triplphasic waveforms of HV. In cirrhotic patients triphasic pattern, biphasic and flat were observed $49.0 \%, 48.0 \%$ and $3.0 \%$ of the patients respectively. Similarly, Bolondi et al. ${ }^{3}$ showed in their study that triphasic pattern was $50.0 \%$, biphasic $31.7 \%$ and flat $18.3 \%$ of the cirrhotic patients. On the other hand Arda et al. ${ }^{10}$ has observed in cirrhotic patients that triphasic pattern was $26.7 \%$, biphasic $60.0 \%$ and flat $13.3 \%$. The result obtained in the present study is a little less than the above mentioned study. This may be due to lack of awareness and delayed health seeking practices in our country.

Herbay et $a^{6}$ observed that the patients with live cirrhosis had regular triphasic (type 1) waveforms occurred significantly less frequently in those with Child $B$ and child $C$ cirrhosis than in those with Child $A$ cirrhosis, which were $60.0 \%$ in Child A, $34.0 \%$ in Child B and $17.0 \%$ in child $C$. Type 2 waveforms occurred in decreasing order of incidence in 
patients with cirrhosis. Type 3 waveforms occurred in decreasing order of incidence in patients with Child C $(66.0 \%)$, child B (49.0\%) and Child A (24.0\%). Similarly, Bolondi et al. ${ }^{3}$ and Sudhamshu et al. ${ }^{2}$ reported the pattern of continuous turbulent HV flow had been more frequent in cirrhotic patients with relatively severer disease as shown by the significant correlation with the Child-Pugh score. In the present series the severity of the liver cirrhosis was assessed by Child-Pugh scoring system with the parameters of encephalopathy, serum bilirubin, serum albumin, prothombin time and ascites. Adding the individual score $<7$ was Child's class A, 7-9 was Child's class B and > 9 was Child's class $C$. It was observed in Child's class A wave form HV-O was found in $75.0 \%, \mathrm{HV}-125.0 \%$ and HV-2 was not found in case group. In Child's class B wave form $\mathrm{HV}-0$ was found in $21.4 \%, \mathrm{HV}-1$ was $71.5 \%$ and $\mathrm{HV}-2$ was $7.1 \%$. In Child's class $\mathrm{C}$ wave form HV-0 was not found, HV-1 was $10.0 \%$ and $\mathrm{HV}-2$ was $90.0 \%$ and the difference was statistically significant $(p<0.05)$ among the different grading level with wave form. The results obtained in the present study are comparable with the above studies.

It was found in the present study according to Child's class $A$ the mean $( \pm S D)$ velocity was $9.18 \pm 2.17 \mathrm{~cm} / \mathrm{sec}$ and their velocity ranged from 6.2 to $15.0 \mathrm{~cm} / \mathrm{sec}$. In Child's class B the mean $( \pm S D$ ) velocity was $11.26 \pm 1.81 \mathrm{~cm} / \mathrm{sec}$ and their velocity ranged from 7.5 to 14.2 $\mathrm{cm} / \mathrm{sec}$. In Child's class $C$ the mean $( \pm S D)$ velocity was $18.44 \pm 191 \mathrm{~cm} / \mathrm{sec}$ and their velocity ranged from 15.5 to $20.9 \mathrm{~cm} / \mathrm{sec}$. The mean velocity was statistically significant $(p<0.05)$ among Child's class A, Child's class $B$ and Child's class $C$.

Sudhamshu et al. ${ }^{2}$ had also made identical observation. According to them the mean $( \pm S D)$ hepatic vein velocity were $9.7 \pm 4.1 \mathrm{~cm} / \mathrm{sec}$ in grade $A, 11.6 \pm 5.4 \mathrm{~cm} / \mathrm{sec}$ in grade $B$, and $19.2 \pm 10.3 \mathrm{~cm} / \mathrm{sec}$ in grade $C$. This result is closely consistent with the present study.

\section{Conclusion}

In this study it was found that Doppler waveform and mean velocity in right hepatic vein is relatively accurate and reproducible. This current study applied to study the right hepatic vein and an attempt has been made to correlate the findings with severity in cirrhotic patients and observed that the cirrhotic patients had increased velocity of the hepatic vein and those cases with very high velocity were having poorer liver function as graded by Child-Pugh classification. On the other hand, the Doppler ultrasound abnormality in hepatic vein waveform could be reasonably considered as an adjunctive sign of liver cirrhosis and are somewhat related to the severity of cirrhosis. Hence, the study advocates that Doppler waveform and measurement of mean hepatic vein velocity, which is non-invasive, can be used as new parameter in the investigation of liver cirrhosis.

\section{Reference}

1. Ong TZ and Tan HJ, "Ultrasonography is not reliable in diagnosing liver cirrhosis in clinical practice", Singapore Med J 2003; 44(6):293-5.

2. Sudhamshu KC, Matsutani S, Maruyama H, Akiike T, Saisho H 2006, "Doppler study of hepatic vein in cirrhotic patients: Correlation with liver dysfunction and hepatic hemodynamics", World J Gastroenterol, 2006 ;12(36): 5853-5858.

3. Bolondi L, Bassi SL, Gaiani S, Zironi G, Benzi B, Santi $V$, Barbara L "Liver cirrhosis: changes of Doppler waveform of hepatic veins1" Radiology 1991;178: 513 . 516.

4. Berry M, Chaudhury V, Mukhopadhyay S, Suri S Editor. Diagnostic Radiology Gastrointestinal and Hepatobiliary Imaging. 2nd edition, Jaypee, 280.

5. Ohta $M$, Hashizume $M$, Tomikawa $M$, Ueno $K$, Tanoue $K$, Sugimachi K, "Analysis of hepatic vein waveform by Doppler ultrasonography in 100 patients with portal hypertension", Am J Gastroenterol, 1994;89:170-175.

6. von Herbay A, Frieling $T$, Haussinger D, "Association between duplex Doppler sonographic flow pattern in right hepatic vein and various liver diseases", $J$ Clin Ultrasound 2001;29:25-30.

7. Colli A, Cocciolo M, Riva C, Martinez E, Prisco A, Pirola M, Bratina G 1994, “Abnormalities of Doppler waveform of the hepatic veins in patients with chronic liver disease: correlation with histologic findings", AJR Am J Roentgenol, 1994;162:833-837.

8. Hayes PC, Simpson KJ, Garden OJ 1995 Liver and Biliary Tract Disease, In: Haslett C, Chilvers ER, Boon NA, Colledge NR. Editor. Davidson's Principles and Practice of Medicine, 17th editioin, Churchill Livingstone: Edingurgh, p. 840.

9. Schuppan D and Afdhal NH 2008, "Liver Cirrhosis",2008 Lancet;371:838-851.

10. Arda K, Ofelli M, Calikoglu U, Olcer $T$ and Gumhur $T$, “ Hepatic vein Doppler waveform changes in early stage (child-pugh A) chronic parenchymal liver disease", $J$ Clin Uitrasound, 1997;25:15-19. 